\title{
Non-Invasive, High-Density Mapping of Human Atrial Fibrillation - Introduction and Illustration of a Novel Diagnostic Tool
}

\author{
MS Guillem ${ }^{1,2}$, AM Climent ${ }^{1,2}$, D Husser $^{1}$, J Millet $^{2}$, A Bollmann ${ }^{1}$ \\ ${ }^{1}$ Otto-von-Guericke Universitäts, Magdeburg, Germany \\ ${ }^{2}$ Universidad Politécnica de Valencia, Valencia, Spain
}

\begin{abstract}
Electrical atrial activation during atrial fibrillation shows an uncoordinated pattern. Invasive studies and computer models have shown that functional and anatomical barriers can sustain atrial fibrillation. Mechanisms involved in the fibrillatory process are multiple wave reentry, rotors and spiral waves.

We have developed a new diagnostic tool with the aim to characterize electrical activation patterns during atrial fibrillation from the body surface. Non-invasive high density mapping using 56 surface electrodes placed on the chest and back of six patients with persistent atrial fibrillation was performed. For the first time, we observed different activation patterns of atrial fibrillation from the body surface: single or multiple wavefronts with different degrees of repeatability.

Further study of the activation patterns could be useful in the diagnosis and individual selection of the best treatment with atrial fibrillation.
\end{abstract}

\section{Introduction}

Atrial fibrillation (AF) is a supraventricular arrhythmia in which the electrical activation shows an uncoordinated pattern. Invasive and modelling studies have shown that this uncoordinated electrical activity can be maintained by anatomical or functional barriers which may provide a substrate for reentry. Rotors, spiral waves and multiple wavelets have been observed in invasive mapping experiments and are thought to be important mechanisms for AF maintenance of [1-4].

Although model studies are of main interest for the understanding of mechanisms underlying AF, they are not directly applicable into clinical practice.

Measurement of the anisotropy in conduction, calculated as the dispersion on the main direction of propagation of electrical wavefronts, has been suggested as a technique for the characterization of complex waveforms that appear in electrograms during AF [5]. Measurement of the anisotropy in conduction appears to be a valuable tool for individual evaluation of the atrial substrate.

Previous non-invasive studies have attempted to evaluate the organization of atrial wavefronts from the vectorcardiogram [6]. However, the use of vectorcardiograms for the $\mathrm{AF}$ characterization can be questioned by the fact that the electrical activity during $\mathrm{AF}$ is sometimes far from being a single wavefront or dipolar.

Surface mapping has been used in the past for detailed characterization of electrical cardiac events from the body surface [7]. This technique has, however, not been applied so far for the characterization of atrial waves during $\mathrm{AF}$. In this study, we present, for the first time, the potential of body surface maps obtained with a custommade lead system for non-invasive evaluation of fibrillatory waves in patients with AF. Further analysis of the activation patterns of each patient can be a noninvasive tool for the characterization of the atrial organization in each individual, thus providing valuable information for the selection of a specific treatment.

\section{Methods}

\section{Population under study}

Six consecutive patients admitted to Otto-vonGuericke University hospital with persistent AF were included in the study.

\section{Acquisition system}

A total of 56 chest and back leads were acquired simultaneously for each subject in addition to the standard limb leads. Chest leads $(\mathrm{N}=40)$ were arranged as a grid around V1 with an inter-electrode distance of 2.2 $\mathrm{cm}$. (See Figure 1) while back leads $(\mathrm{N}=16)$ were arranged in a similar fashion around V1post (placed on the back at the same level than V1).

We designed a new belt for specifically attaching the electrodes in the correct position on the chest and back of the patient. Perforations are applied to the belt according 
to the electrode grid designed. Elastic bands cover the perforations providing fixation of the electrodes and applying the needed pressure. The front and back parts of the belt are joined with stripes of adjustable length, adapting to the shape of the patient. This attachment system allows repeatability in the measurements and provides a good contact of the electrodes with the skin of patient, reducing movement artefacts.

Signals were acquired at a sampling rate of $2048 \mathrm{~Hz}$, with a resolution of 1 microvolt and a bandwidth of 500 Hz. Before acquisition, signal quality of all leads was visually inspected and an ECG recording of 10 minutes was stored for off-line processing.

\section{ECG Signal Processing}

ECG signals were processed using Matlab 7.0.1 (The Mathworks Inc, The Neatherlands). Baseline wandering was reduced by subtracting the baseline to the recording, calculated by using a $3^{\text {rd }}$ order low-pass Chebyshev filter. Signals were low-pass filtered in order to avoid myoelectric interference by using a low-pass Butterworth filter $(\mathrm{fc}=40 \mathrm{~Hz})$ and downsampled to $512 \mathrm{~Hz}$ in order to speed up the calculations.

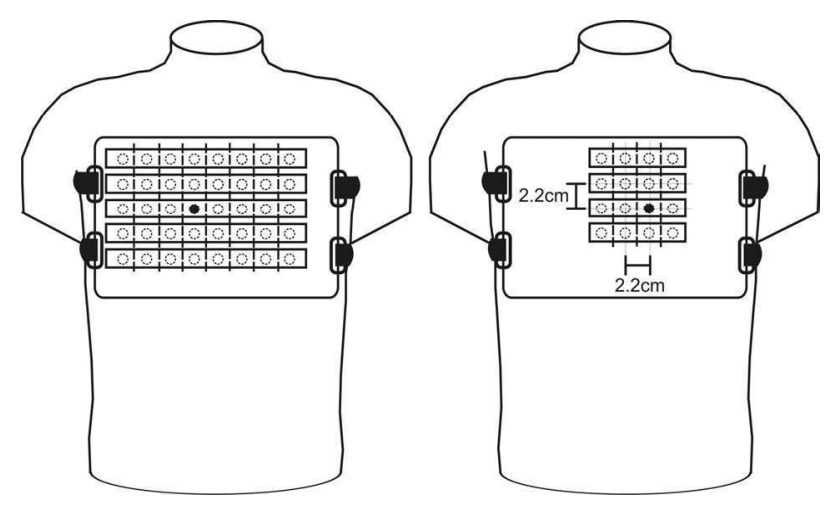

Figure 1: Arrangement of the electrodes and belt used for their attachment to the patient. Electrode positions are represented as open circles while V1 and V1post are denoted by black circles. The rest of electrodes were placed around V1 and V1post as a uniform grid.

Only long segments free from ventricular content were included in the study. For that purpose, the peaks of QRS complexes were detected in V1. RR intervals longer than $950 \mathrm{~ms}$ were first selected and a TQ segment was defined as starting $400 \mathrm{~ms}$ after the QRS peak and ending $150 \mathrm{~ms}$ before the next QRS peak. All TQ segments were visually inspected in order to avoid wrong detections or inclusion of $\mathrm{T}$ segments. After this step, only segments free from ventricular activity and longer than $400 \mathrm{~ms}$ were considered.
Typically, one to three leads presented noticeable baseline wandering which was comparable or greater than the amplitude of the AF signal. In order to avoid this baseline wandering, AF segments were filtered again. First, the mean value in each lead was subtracted and the baseline was again calculated and subtracted. For the estimation of the baseline, segments were downsampled ( $\mathrm{fs}=128 \mathrm{~Hz}$ ) and low-pass filtered $(\mathrm{fc}=3.3 \mathrm{~Hz}$ ). Then, the baseline was upsampled to $512 \mathrm{~Hz}$ and subtracted to the segment. After baseline wandering correction, AF segments were low-pass filtered $(\mathrm{fc}=20 \mathrm{~Hz})$.

All leads in all segments were visually inspected. Leads presenting noticeable noise contributions (typically, a transient loss of contact in one electrode) were discarded and interpolated from its neighbouring electrodes by cubic spline interpolation. No segment presented more than three discarded leads, and we considered that the loss of signal content of less than 4 electrodes out of the 56 available was acceptable.

Once AF segments had been isolated and processed, maps for each time instant belonging to the each segment were constructed. For that purpose, potentials at a given time instant were arranged in two matrices: one for the front $(5 \times 8)$ and one for the back $(4 \times 4)$. In such matrices, signals recorded from neighbouring electrodes are placed together. In order to obtain a smooth representation of the maps, finer matrices for the front $(50 \times 80)$ and the back $(40 \mathrm{x} 40)$ were created. Potentials for unknown positions were interpolated by cubic spline interpolation.

Two display modes were implemented: the isopotential mode and the wavefront propagation mode. In the isopotential mode, voltages at a given time instant were represented according to a color scale. Videos of each AF segment were generated. In the wavefront propagation mode, only lines connecting points on the surface with a voltage equals to zero were represented. This display mode allows the visualization of both the depolarization and repolarization wavefronts depending on the time interval selected. The succession of wavefronts is represented using a color scale according to the time instant in which each wavefront appears.

\section{Results}

Maps were generated for all the segments considered in the study and visually inspected. Single or multiple simultaneous wavefronts, wave breakages and disorganized electrical activity could be observed.

Figure 2 shows some selected wavefront propagation maps representative patterns observed.

In panel 1a, a succession of wavefronts from patient 3 is observed. A single wavefront can be observed in both panels $1 \mathrm{a}$ and $1 \mathrm{~b}$. In panel 1a wavefronts are mostly parallel to each other. Panel $1 \mathrm{~b}$ shows the next succession of wavefronts, which differ from panel 1a. 

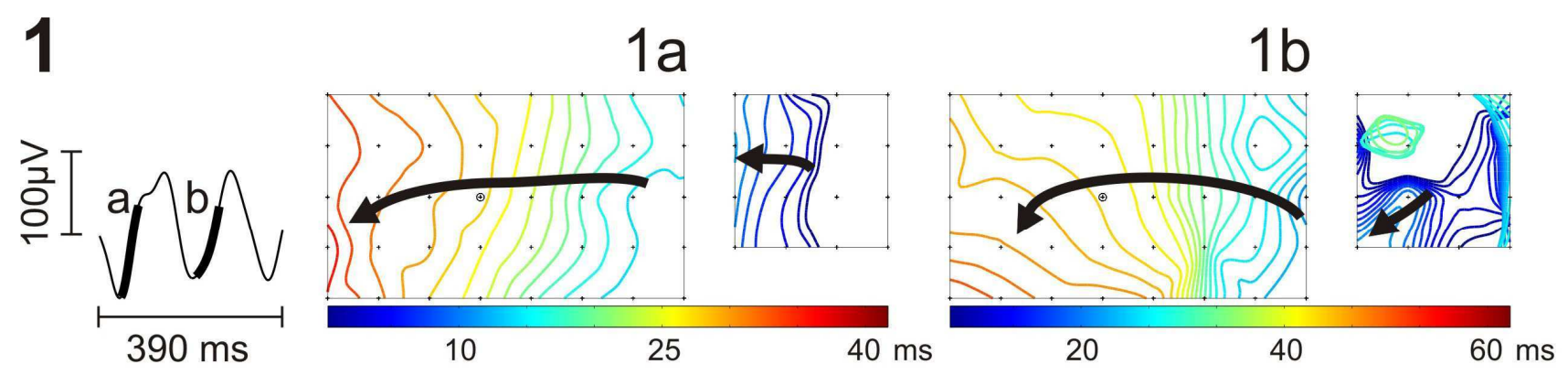

2

$2 a$

$2 b$
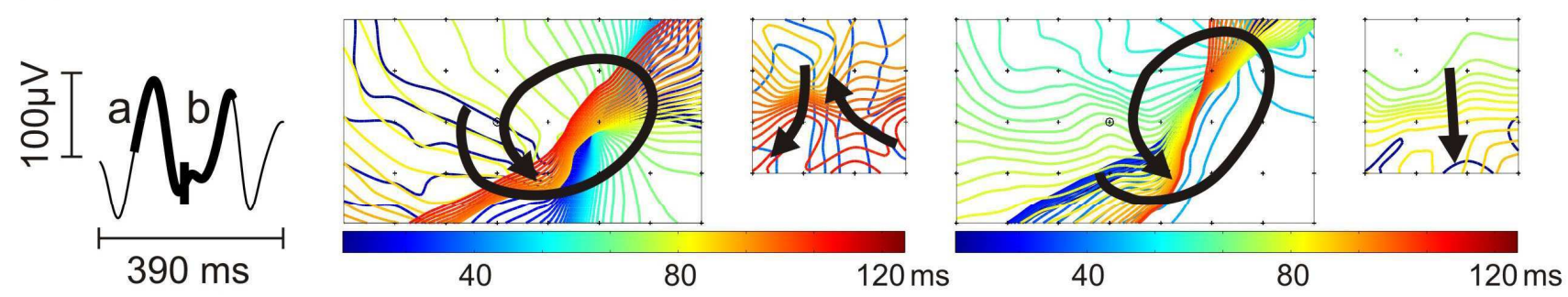

3

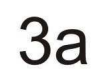

$3 b$
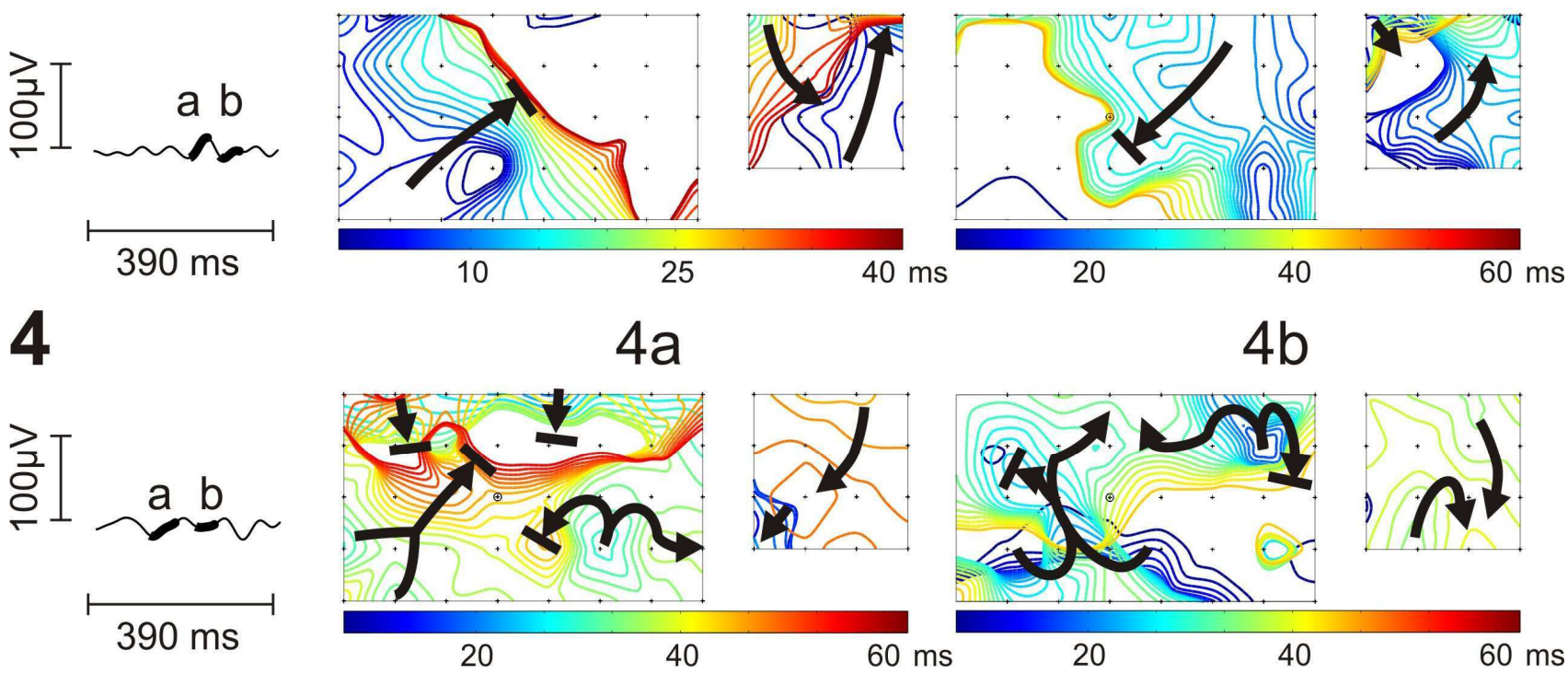

Figure 2: Wavefront propagation maps of four selected AF segments. On the left, tracings corresponding to V1 for the segments chosen. Panels a and b correspond to wavefront propagation maps of two intervals of the same segment in a color scale. The segments represented in panels $\mathrm{a}$ and $\mathrm{b}$ are denoted by heavier lines in the tracings. On the left in each panel, maps corresponding to the front part of the thorax. On the right, maps corresponding to the back. Each electrode position is labelled with a '+' sign. V1 is represented by a circle. Wavefront propagation lines are drawn every $2 \mathrm{~ms}$. According to the color scale, wavefronts appearing first are drawn in blue, while wavefronts appearing last are drawn in red. Notice that the color scale is different in each figure according to the length of the interval selected. Arrows indicate the direction of propagation of each wavefront. Panels 1 and 2 show two AF segments from patient 3 while panels 3 and 4 represent AF segments from patients 5 and 6 respectively. 


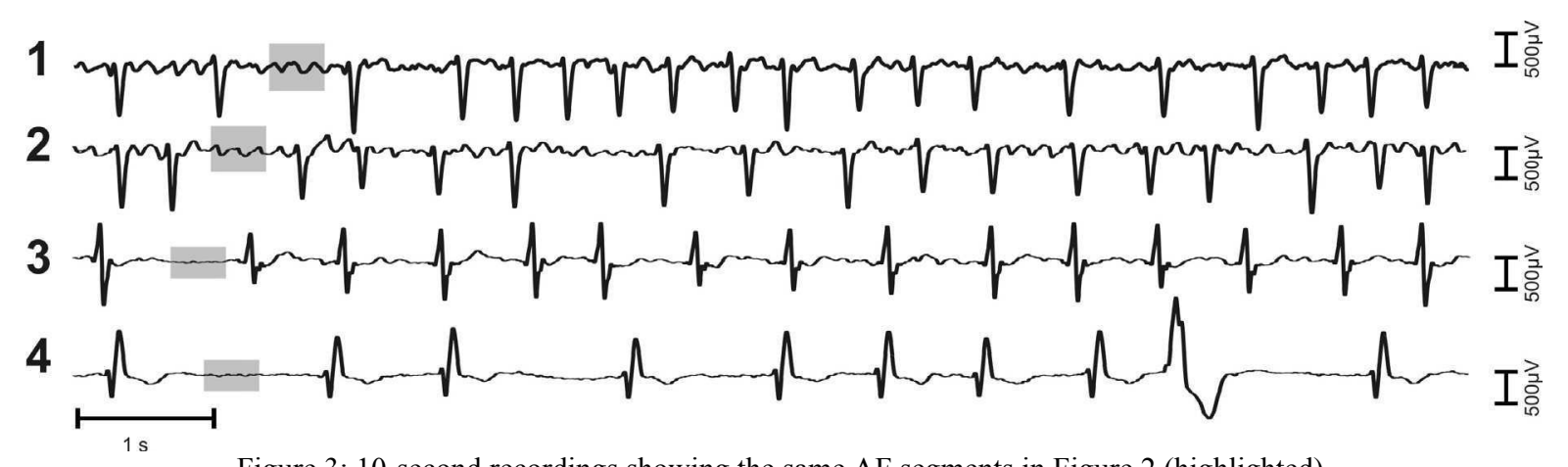

Figure 3: 10-second recordings showing the same AF segments in Figure 2 (highlighted).

Panels $2 \mathrm{a}$ and $2 \mathrm{~b}$ show two consecutive depolarization waves from patient 3 which present a similar pattern. Surface wavefronts rotate along a similar axis in both panels. The same pattern was observed in the consecutive wavefronts appearing in the same segment (total $=5$ ).

Panel 3 shows two consecutive depolarization waves from patient 5. Depolarization wave shown in panel a is first shown in the leftmost part on bottom of the grid and spreads towards the center and top until it gets blocked. A second depolarization wave starts in the uppermost part on the right of the grid and propagates towards the bottom and the left until it gets blocked in roughly the same position where the previous wave arrived. It can be observed that one wavefront is blocked by the previous propagation contributing to a chaotic propagation.

Panels $4 \mathrm{a}$ and $4 \mathrm{~b}$ show depolarization waves in patient 6. Multiple waves arise at the same time, propagate and then disappear in an uncoordinated fashion.

By analyzing the 10-minute recording of each patient, we observed that the wavefront propagation patterns were, to some extent, repeatable. Patient 3 showed clear wavefronts with similar patterns in most segments analyzed. Patients 1, 5 and 6 showed a completely unstable pattern with multiple simultaneous activation wavefronts that changed widely even in short time intervals. Patients 2 and 4 presented an intermediate degree of repeatability, with one or two simultaneous propagation wavefronts that could both differ greatly or slightly from one activation to another.

\section{Discussion and conclusions}

A non-invasive method for the study of electrical activation in AF has been presented. Different patterns of electrical activation and degrees of stability of the activation patterns have been observed.

Further quantification of the propagation wavefronts appears as a promising tool for the study of the degree of organization in the atria with AF.

The comparison of this new method for the quantification of the degree of organization of the atrial electrical activity with other methods such as spectral analysis and validation by comparison with invasive mapping data will be of great interest in future studies.

Although observations from the present study have not been compared to the effectiveness of any specific treatment, there is a great potential in further analyzing the activation patterns of the electrical wavefronts occurring in the atria as observable in body surface maps.

\section{Acknowledgements}

This work was partially supported by the grants UPV, ENFASIS-TEC2005-08401 (Spain) and the Volkswagen Foundation (Germany).

\section{References}

[1] Jalife J. Rotors and spiral waves in atrial fibrillation. J Cardiovasc Electrophysiol 2003; 14:776-780.

[2] Skanes A, Mandapati R, Berenfeld O, Davidenko JM et al. Spatiotemporal Periodicity During Atrial Fibrillation in the Isolated Sheep Heart. Circulation 1998; 98: 1236-1248.

[3] Reumann M, Bohnert J, Osswald B, Hagl S and Doessel O. Multiple wavelets, rotors and snakes in atrial fibrillation -a computer simulation study. J Electrocardiol 2007. In Press

[4] Jacquemet V, Van Oosterom A, Vesin JM, Kappenberger L. Analysis of Electrocardiograms During Atrial Fibrillation. IEEE Engineering in Medicine and Biology 2006; 25(6): 79-88.

[5] Houben R and Allessie M. Processing of Intracardiac Electrograms in Atrial Fibrillation. IEEE Engineering in Medicine and Biology 2006; 25(6): 40-51.

[6] Ng J, Sahakian AV, Fisher WG and Swiryn S. Surface ECG vector characteristics of organized and disorganized atrial activity during atrial fibrillation. J Electrocardiol 2004; 37:91-97.

[7] Bodi V, Sanchis J, Guillem MS et al. Analysis of the extension of Q-waves after infarction with body surface map: Relationship with infarct size. International Journal of Cardiology 2006; 111: 399-402

Address for correspondence

María de la Salud Guillem Sánchez

BET, ITACA, Ciudad Politécnica de la Innovación

Camino de Vera s/n 46022 Valencia, Valencia, Spain 